\title{
Trauma, Resilienz und Emotionsregulation
}

\author{
Trauma, Resilience, and Emotion Regulation
}

\author{
Autoren \\ Julia Holl1 ${ }^{1}$, Isabel Pap ${ }^{1}$, Jonna Südhof ${ }^{2}$, Elisabeth Wolff', \\ Katharina Staben ${ }^{1}$, Sebastian Wolff' ${ }^{1}$, Anja Höcker ${ }^{3,4}$, Philipp Hiller ${ }^{3,4}$, \\ Ingo Schäfer ${ }^{3,4}$, Sven Barnow ${ }^{1}$ \\ Institute \\ 1 Klinische Psychologie und Psychotherapie, Psychologisches \\ Institut, Ruprecht-Karls-Universität Heidelberg \\ 2 Zentralinstitut für seelische Gesundheit, Zentrum für Psychologi- \\ sche Psychotherapie (ZPP), Mannheim \\ 3 Klinik für Psychiatrie und Psychotherapie, Universitätsklinikum \\ Hamburg-Eppendorf \\ 4 Zentrum für Interdisziplinäre Suchtforschung (ZIS), Universität \\ Hamburg
}

Schlüsselwörter

traumatische Erfahrungen in der Kindheit und Jugend, Psychopathologie, Emotionsregulation, Resilienz, gesunde Personen

Keywords

childhood abuse and neglect, psychopathology, emotion regulation, resilience, healthy individuals

eingereicht 2.05.2016

akzeptiert 13.10.2016

Bibliografie

DOI http://dx.doi.org/10.1055/s-0042-120271

Psychother Psych Med 2017; 67: 83-90

(c) Georg Thieme Verlag KG Stuttgart · New York

ISSN 0937-2032

Korrespondenzadresse

Dr. phil. Julia Holl, Dipl.-Psych.

Ruprecht-Karls-Universität Heidelberg

Psychologisches Institut

Klinische Psychologie und Psychotherapie

Hauptstraße 47-51

69117 Heidelberg

julia.holl@psychologie.uni-heidelberg.de

\section{ZUSAMMENFASSUNG}

Einleitung: Das Erleben traumatischer Erfahrungen in der Kindheit und Jugend gilt als Risikofaktor für Psychopathologie. Eine funktionale Emotionsregulation (ER) könnte für einen späteren resilienten Entwicklungsverlauf bedeutsam sein.

Material \& Methoden: In dieser Studie wurde der habituelle Einsatz von spezifischen ER-Strategien zwischen psychisch gesunden Personen mit $(n=61)$ und ohne $(n=52)$ traumatische Erfahrungen in der Kindheit und Jugend mithilfe des Heidelberger Fragebogen zur Erfassung der Emotionsregulationsstrategien (H-FERST) verglichen. Psychopathologie wurde mittels SKID-I, ADP-IV, SCL-27 und BDI-II erfasst.

Ergebnisse: Die beiden Gruppen unterschieden sich nicht signifikant im habituellen Einsatz der ER-Strategien. Die gesunden Personen mit traumatischen Erfahrungen gaben eine signifikant höhere subjektive psychische Belastung an.

Diskussion \& Schlussfolgerung: Vor dem Hintergrund einer subjektiv höheren psychischen Belastung könnte eine funktionale habituelle Nutzung von ER-Strategien einen Erklärungspfad für die resiliente Entwicklung von Betroffenen mit traumatischen Erfahrungen in der Kindheit und Jugend darstellen.

\section{ABSTRACT}

Background: History of childhood abuse and neglect is considered to be a relevant risk factor for adult psychopathology. A functional emotion regulation (ER) can account for resilience despite of traumatic experiences in childhood.

Materials \& Methods: This study compares the habitual use of specific ER strategies among mentally healthy individuals with $(n=61)$ and without ( $n=52$ ) experience of childhood abuse and neglect by using the self-rating instrument Heidelberg Form for Emotion Regulation Strategies (H-FERST). SCID-I, ADP-IV, SCL-27, and BDI-II were used for assessment of psychopathological distress.

Results: We found no group difference in the habitual use of ER strategies. Healthy individuals with childhood abuse and neglect showed significantly more subjective distress symptoms.

Discussion \& Conclusion: Considering the significantly higher psychopathological distress reported by the trauma group, the functional habitual use of ER strategies could serve as a path to explain the resilient development of adult individuals after childhood abuse and neglect.

\section{Einleitung}

Aktuellen Zahlen zufolge ist das Erleben von Missbrauchs- und Vernachlässigungserfahrungen im Kindes- und Jugendalter (sexueller, physischer, emotionaler Missbrauch sowie physische, emotionale Vernachlässigung) mit einer Prävalenz von 33,9\% in Deutschland weit verbreitet [1]. Dabei handelt es sich zumeist um sogenannte Typ-II-Traumata - wiederholt auftretende oder anhaltende interpersonelle traumatische Erfahrungen [2] - die mit schwerwiegenden und komplexen Auswirkungen für die psychische Gesundheit im Erwachsenenalter assoziiert sind [3].

Gleichzeitig weisen ca. 30\% der Betroffenen im Erwachsenenalter eine adaptive Lebensführung auf [4]. Eine mögliche Erklärung hierfür liefert das Konzept der Resilienz, das einen erfolgreichen Anpassungsprozess infolge schwerer Belastungen beschreibt [5]. So gilt eine Person als resilient, wenn sich nach dem Erleben von potenziell traumatisierenden Ereignissen keine psychopathologische Störung diagnostizieren lässt, sich anfängliche subsyndromale posttraumatische Reaktionen auflösen oder sich ein höheres Funktionsniveau beobachten lässt. Die Prozesse einer erfolgreichen Anpassung in Situationen mit hoher Belastung können auf eine Vielzahl psychologischer, biologischer, interpersoneller und soziokultureller Faktoren zurückgeführt werden [6].

Unterschiede in der Emotionsregulation (ER) können sowohl einen resilienten als auch einen psychopathologischen Entwick- 
lungsverlauf nach dem Erleben von frühen traumatischen Erfahrungen erklären. Empirisch ist das Erleben früher traumatischer Erfahrungen assoziiert mit ER-Defiziten, wie der reduzierten Fähigkeit emotionale Zustände zu differenzieren [7]. ER-Defizite wiederum erhöhen das Risiko für eine psychopathologische Entwicklung [8]. Gleichzeitig kann eine effektive und funktionale ER für die Aufrechterhaltung der psychischen Gesundheit relevant sein [9]. Bei der Untersuchung von Personen, die traumatische Erfahrungen erlebt haben, hat sich der emotionale Umgang mit diesen Erfahrungen als bedeutsamer für interindividuelle Resilienzunterschiede erwiesen als der Schweregrad der erlebten traumatischen Erfahrungen [10]. Demnach könnte die Untersuchung der Variabilität emotionaler Prozesse nach frühen traumatischen Erfahrungen zu einem besseren Verständnis der unterschiedlichen Entwicklungsverläufe beitragen.

Laut Gross' theoretischer Konzeptualisierung bezieht sich ER auf die Prozesse, durch die Individuen beeinflussen, welche Emotionen sie haben, wann sie diese haben und wie sie diese erleben und ausdrücken [11]. ER umfasst somit die Wahrnehmung und Regulation sowohl negativer als auch positiver Emotionen. Dem Einsatz verschiedener ER-Strategien, wie Rumination, Neubewertung, Akzeptanz, Problemlösen, Suppression, Vermeidung, Aktivität und soziale Unterstützung sowie Ablenkung, wird v. a. bei der Regulation negativer Emotionen eine wichtige Rolle zugeschrieben [12]. Zudem wird im Zusammenhang mit Psychopathologie zwischen adaptiven und maladaptiven ER-Strategien unterschieden: Adaptive Strategien begünstigen eine effektive und funktionale ER, insofern dass ihr Einsatz zu einer Reduzierung der erlebten negativen Emotionen führt und dabei die kurz- und langfristigen Ziele eines Individuums unbeeinträchtigt bleiben. Der Einsatz maladaptiver Strategien hingegen kann eine ineffektive und dysfunktionale ER begünstigen, indem entweder die unerwünschte Emotion nicht reduziert wird oder langfristige Kosten den kurzfristigen Nutzen der verminderten Emotion überwiegen [13].

Die ER-Strategien Neubewertung, Akzeptanz und Problemlösen können als langfristig adaptiv verstanden werden, da ihr Einsatz mit weniger psychopathologischen Symptomen assoziiert ist [14]. Rumination, Suppression und Vermeidung erwiesen sich hingegen als langfristig maladaptive Strategien, die entsprechend in höherem Maße positiv mit Psychopathologie assoziiert waren [14]. Die Nutzung von adaptiven ER-Strategien könnte demnach als Schutzfaktor und der Einsatz von maladaptiven ER-Strategien als Risikofaktor für Psychopathologie verstanden werden.

Empirisch findet sich ein Zusammenhang zwischen dem Erleben traumatischer Erfahrungen und der dysfunktionalen Regulation von negativen Emotionen dergestalt, dass traumatisierte Personen im Vergleich zu gesunden Personen ohne traumatische Erfahrungen mehr und intensivere negative Emotionen wahrnahmen [15]. Demnach könnten Missbrauchs- und Vernachlässigungserfahrungen einen Einfluss auf die Fähigkeit für eine funktionale ER haben [16]. Unter dieser Annahme dürfte einer effektiven Reduzierung negativer Emotionen und damit der funktionalen Nutzung von ER-Strategien insbesondere bei traumatisierten Personen eine wichtige Funktion zur Aufrechterhaltung der psychischen Gesundheit zukommen. Gleichzeitig gibt es auch Hinweise auf positive Folgen traumatischer Erfahrungen (posttraumatic growth), wie ein höheres psychosoziales Funktionsniveau [17]. Folglich könnte das
Erleben traumatischer Erfahrungen auch zu einer effektiveren und funktionaleren ER beitragen, da die Betroffenen in der Vergangenheit stärker darauf angewiesen waren bzw. noch sind, ihre Emotionen effektiv zu regulieren.

Allerdings fokussiert die empirische Forschung zu Auswirkungen von frühen traumatischen Erfahrungen schwerpunktmäßig auf klinische Stichproben, deren Befunde nur eine begrenzte Aussage über psychische Prozesse von gesunden Personen mit einem Traumahintergrund zulassen [18]. Durch diese defizitorientierte Perspektive wird die Untersuchung von Ressourcen vernachlässigt, deren Kenntnis für die Entwicklung von Präventionsmaßnahmen zur Aufrechterhaltung der psychischen Gesundheit nach dem Erleben früher traumatischer Erfahrungen bedeutsam sein könnte.

Insgesamt ist also die Untersuchung der ER bei psychisch gesunden Personen, die traumatische Erfahrungen in der Kindheit und Jugend gemacht haben, weit unterrepräsentiert. Diese Personen stellen aufgrund der frühen traumatischen Erfahrungen eine Hochrisikostichprobe für Psychopathologie dar und weisen gleichzeitig einen resilienten Entwicklungsverlauf auf. Die Untersuchung der ER dieser Stichprobe gibt damit potenziell Hinweise darauf, wie Personen nach dem Erleben traumatischer Erfahrungen in der Kindheit und Jugend psychische Gesundheit aufrechterhalten können. Zusätzlich kann die Untersuchung der ER von psychisch gesunden Personen, die keine frühen traumatischen Erfahrungen gemacht haben, als Referenz für normative ER-Prozesse dienen, sodass die vergleichende Untersuchung der Nutzung von ER-Strategien lohnenswert sein könnte.

Ziel der vorliegenden Studie ist es deshalb, die habituelle Nutzung von 7 spezifischen und 2 verhaltensorientierten ER-Strategien zwischen psychisch gesunden Personen mit traumatischen Erfahrungen im Kindes- und Jugendalter (Traumagruppe) und gesunden Kontrollprobanden ohne solche Erfahrungen (Kontrollgruppe) mittels Heidelberger Fragebogen zur Erfassung von Emotionsregulationsstrategien (H-FERST; [12]) zu vergleichen. Die Beschäftigung mit dieser kaum untersuchten Fragestellung erfolgt erstmalig in einem querschnittlichen und retrospektiven Design, um Hinweise zu erhalten, inwieweit eine funktionale Nutzung von ER-Strategien in diesem Zusammenhang bedeutsam sein könnte. Die Personen der Traumagruppe haben in ihrer Vorgeschichte frühe traumatische Erfahrungen gemacht, aber weisen sowohl aktuell als auch über die bisherige Lebensspanne hinweg psychische Gesundheit auf, was eine vorsichtige längsschnittliche Interpretation der Befunde zulassen könnte und damit einen Erklärungspfad für die resiliente Entwicklung nach dem Erleben traumatischer Erfahrungen in der Kindheit und Jugend darstellen könnte.

Ausgehend von der inkonsistenten empirischen Datenlage kann nicht eindeutig vorhergesagt werden, ob die Traumagruppe verglichen mit der Kontrollgruppe einen funktionaleren, einen ähnlich ausgeprägten oder einen dysfunktionaleren habituellen Einsatz der ER-Strategien aufweist. Als ein funktionales ER-Profil definieren wir einen häufigeren Einsatz von adaptiven (Neubewertung, Problemlösen, Akzeptanz, Aktivität und soziale Unterstützung) und selteneren Einsatz maladaptiver Strategien (Rumination, Unterdrückung des emotionalen Ausdrucks, Unterdrückung des emotionalen Erlebens, Vermeidung, Ablenkung). Bei einem dysfunktionalen ER-Profil werden entsprechend die maladaptiven ER-Strategien häufiger und die adaptiven seltener eingesetzt. 


\section{Material und Methoden} Stichprobe und Studiendesign

Die vorliegende Untersuchung ist Teil des vom Bundesministerium für Bildung und Forschung (BMBF) geförderten Verbundprojekts CANSAS (Akronym für childhood abuse and neglect as a cause and consequence on substance use abuse - understandig risks and improving services) (BMBF: 01KR1203B) zur Identifikation von Risiko- und Schutzfaktoren für eine Substanzstörung vor dem Hintergrund traumatischer Erfahrungen in der Kindheit und Jugend.

Generelle Einschlusskriterien der Probanden für die vorliegende Untersuchung waren 1) Alter zwischen 18 und 65 Jahren und 2) ausreichende deutsche Sprachkenntnisse. Generelle Ausschlusskriterien waren 1) der Verdacht auf eine aktuelle oder frühere vorliegende Achse-I- oder Achse-II-Störung, 2) neurologische Erkrankungen und 3) schwerwiegende kognitive Beeinträchtigungen. Zusätzlich galt ein gruppenspezifisches Einschlusskriterium bezüglich traumatischer Erfahrungen in der Kindheit und Jugend, was durch Cut-off-Werte des Childhood Trauma Questionnaires (CTQ; [19]) bestimmt wurde. Personen konnten in die Traumagruppe $(n=61)$ aufgenommen werden, bei einer mindestens moderaten bis schweren Ausprägung von mindestens einer der bekannten Formen von emotionalem, physischem, sexuellem Missbrauch und/oder emotionaler, physischer Vernachlässigung in der Vergangenheit. Personen konnten in die Kontrollgruppe $(n=52)$ eingeschlossen werden, wenn sie weder traumatische Erfahrungen in der Kindheit und Jugend noch im Erwachsenenalter erlebt hatten.

Die Probandenrekrutierung erfolgte im Raum Heidelberg und Hamburg über öffentliche Medien (Probandenaufrufe durch Zeitungsartikel, Radio, Internet), E-Mail-Verteiler ansässiger Unternehmen sowie Aushänge und Flyer an öffentlichen Institutionen (Universitäten, Krankenhäuser usw.). Zur Überprüfung der Ein- bzw. Ausschlusskriterien erfolgte zunächst ein 2-stufiges ca. 2-stündiges Screeningverfahren. Die nachfolgende fragebogen- und interviewbasierte Untersuchung wurde in standardisierter Reihenfolge und pseudonymisierter Form durchgeführt und beanspruchte ca. 2 Stunden. Die Datenerhebung erfolgte durch qualifiziertes Personal (mind. B.-Sc.-Abschluss in Psychologie), das im Vorfeld eine entsprechende Schulung erhielt. Darüber hinaus fanden regelmäßig Teamintervisionen zur Entscheidung über Studienein - bzw. ausschluss der Probanden statt.

Von initial $n=205$ gescreenten Personen konnten $n=113$ in die Untersuchung eingeschlossen werden, deren Daten vollständig vorlagen. Der Ausschluss von potenziellen Probanden erfolgte hauptsächlich aufgrund psychopathologischer Auffälligkeiten, insbesondere dem Verdacht auf depressive Erkrankungen und Substanzmissbrauch. Die Studie wurde von der Ethikkommission der Ruprecht-Karls-Universität Heidelberg und der Ärztekammer Hamburg bewilligt. Nach Aufnahme in die Studie erteilten die Probanden schriftlich ihre Einverständniserklärung und erhielten eine Aufwandsentschädigung (finanziell bzw. Versuchspersonenstunden).

\section{Messinstrumente}

Die Kurzversion des CTQ ist ein reliables, valides, ökonomisches und international häufig eingesetztes Selbstbeurteilungsinstrument für die retrospektive Erfassung des Vorliegens und des Schweregrads von traumatischen Erfahrungen in der Kindheit und Jugend [20].
Der CTQ besteht aus 28 Items, die die 5 Subskalen emotionalen, physischen und sexuellen Missbrauch sowie emotionale und physische Vernachlässigung bilden. 3 Zusatzitems erfassen Bagatellisierungseffekte [19]. Die 5-minütige Bearbeitung erfolgt auf einer 5-stufigen Likert-Skala ( 1 = überhaupt nicht; 5 = sehr häufig). Für die Summenwerte der Skalen existieren Cut-off-Werte der Originalautoren [19], die eine Schweregradeinteilung der jeweiligen Subskala in „nicht bis minimal“, „niedrig bis moderat“, „moderat bis schwer“ und „schwer bis extrem“ ermöglichen. Zur Auswertung wurden die Summenwerte für die 5 Subskalen berechnet. Höhere Werte reflektieren ein größeres Ausmaß an erlebten traumatischen Erfahrungen im Kindes- und Jugendalter. Die deutschsprachige Kurzversion des CTQ zeichnet sich durch gute psychometrische Kennwerte aus. Cronbach's Alpha beträgt $\geq 0,94$ für die einzelnen Skalen; ihre Konstruktvalidität liegt im akzeptablen Bereich [20].

Der Heidelberger Fragebogen zur Erfassung der Emotionsregulationsstrategien (H-FERST; [12]) erfasst den habituellen Einsatz von ER-Strategien. Dieses sich im deutschsprachigen Raum in Validierung befindliche Selbstbeurteilungsinstrument besteht aus 47 Items und umfasst 7 spezifische ER-Strategien - Rumination, Neubewertung, Akzeptanz, Problemlösen, Unterdrückung des emotionalen Ausdrucks, Unterdrückung des emotionalen Erlebens, Vermeidung - sowie 2 verhaltensorientierte Strategien - Aktivität und soziale Unterstützung, Ablenkung. Die Probanden schätzten auf einer 5-stufigen Likert-Skala ein, inwieweit die jeweiligen Aussagen typischerweise auf sie zutreffen ( 1 = trifft gar nicht zu; 5 = trifft zu). Die Auswertung der jeweiligen ER-Strategien erfolgt über die Summenwerte der Items. Erste Befunde der Normierungsstichprobe deuten bei den spezifischen ER-Strategien auf eine gute $(\alpha>0,81)$ und bei den verhaltensorientierten Strategien auf eine akzeptable interne Konsistenz $(0,6<\alpha<0,7)$ hin. Des Weiteren ließen sich 2 übergeordnete Faktoren für adaptive Strategien (Neubewertung, Problemlösen, Akzeptanz, Aktivität und soziale Unterstützung) und maladaptive Strategien (Grübeln, Vermeidung, z. T. Ablenkung) extrahieren. Ein dritter Faktor umfasst die 2 Strategien zur Unterdrückung des emotionalen Erlebens bzw. Ausdrucks [12].

Die Forschungsversion des Strukturierten Klinischen Interview zur Erfassung psychischer Störungen nach DSM-IV: Achse I (SKIDI) diente dazu, dass Vorliegen einer aktuellen oder vergangenen Achse-I-Störung auszuschließen [21]. Dieses halbstrukturierte Interview berücksichtigt alle psychischen Störungen und Syndrome der Achse-I nach DSM-IV und verfügt über befriedigende psychometrische Eigenschaften [21].

Die deutsche Version des Beck Depressionsinventar (BDI-II; [22]) diente der Erfassung depressiver Beschwerden innerhalb der letzten 2 Wochen. Das Selbstbeurteilungsinstrument besteht aus 21 Items, die auf einer 4-stufigen Likert-Skala ( 0 -3) beantwortet werden. Die Auswertung erfolgt über den Summenwert. Höhere Werte indizieren einen größeren Schweregrad depressiver Symptome. Der Fragebogen weist eine gute Reliabilität (Cronbach's Alpha = $0,84)[23]$.

Die deutsche Version der Symptom-Checkliste SCL-27 wird häufig als ein Screening der allgemeinen Psychopathologie eingesetzt [24]. In 53 Items werden für die vergangenen 7 Tage auf einer 5-stufigen Likert-Skala ( 1 = überhaupt nicht; 5 = sehr stark) 6 Subskalen (depressive, dysthyme, vegetative, agoraphobische, soziophobische sowie Symptome von Misstrauen) abgebildet. Der glo- 
bale Schwereindex (CSI-27), der sich aus dem Mittelwert über alle Items zusammensetzt, wurde zur Auswertung herangezogen. Höhere Werte indizieren eine schwerer ausgeprägte psychopathologische Belastung. Die internen Reliabilitätswerte des GSI-27 sind exzellent (Cronbach's Alpha =0,93) [24].

Die deutsche Version des Assessment of DSM-IV Personality Disorders Questionnaire (ADP-IV; [25]) wurde zur kategorialen und dimensionalen Erfassung der 12 im DSM-IV aufgeführten Persönlichkeitsstörungen eingesetzt. Die 20-minütige Bearbeitung der insgesamt 94 Items des Selbstbeurteilungsinstruments erfolgt über eine 7-stufige Likert-Skala (1-7), wobei höhere Werte eine größere Übereinstimmung mit dem jeweiligen Item indizieren.Weiterhin wurde für positiv beurteilte Items auf einer 3-stufigen Skala (1-3) die subjektive Belastung, die der betreffende Persönlichkeitszug für die Versuchsperson darstellt, in die Beurteilung einbezogen. Zur Auswertung diente der dimensionale Score aufgrund einer überlegenen Validität gegenüber der kategorialen Diagnostik [25]. Dies bietet zudem die Möglichkeit zur Einschätzung subklinischer Traits. Der ADP-IV weist zufriedenstellende psychometrische Eigenschaften auf. Das mittlere Cronbach's Alpha für die einzelnen Subskalen liegt bei 0,76 (Range 0,65-0,87) [25].

\section{Statistische Auswertung}

Die Datenauswertung erfolgte mit der Statistik-Software IBM SPSS Statistics (Version 20.0). Alle Teststatistiken wurden 2-seitig unter $\operatorname{der}$ Annahme von $\alpha=0,05$ berechnet. Das Signifikanzniveau wurde bei Berechnungen mit mehreren abhängigen Variablen durch die Bonferroni-Korrektur angepasst. Bei den nominalskalierten Variablen erfolgte die Berechnung von Chi-Quadrat-Tests zur Prüfung statistisch relevanter Gruppenunterschiede. Zur Prüfung der Unterschiedshypothesen bei den intervallskalierten Variablen diente die Berechnung 2-seitiger $t$-Tests für unabhängige Stichproben. Bei Verletzung der Normalverteilungsannahme wurde ein non-para- metrisches Verfahren - Mann-Whitney-U-Test - herangezogen. Schließlich ermöglichte die Berechnung von Univariaten Kovarianzanalysen (ANCOVAs) die Kontrolle potenzieller Störvariablen. Bei der Analyse der ER-Strategien zwischen den Studiengruppen wurde die psychopathologische Belastung als Kovariate aufgenommen, um einen potenziell konfundierenden Effekt zu kontrollieren. Zudem wurden Effektstärken für die jeweiligen Verfahren berechnet (Cohen's d, Cohen's $\omega$, Eta-Quadrat $\left(\eta^{2}\right)$ ).

\section{Ergebnisse \\ Soziodemografische Angaben}

Die Stichprobe befand sich im mittleren Lebensalter ( $M=35,5$ Jahre, $S D=13,92)$, war überwiegend weiblich (69\%), deutscher Nationalität (85\%) und berufstätig bzw. befand sich in Ausbildung/Studium (94\%). $86 \%$ der Probanden verfügte über eine Hochschulreife und ein Großteil befand sich zum Untersuchungszeitpunkt in einer festen Beziehung (67\%). Es fanden sich keine bedeutsamen Gruppenunterschiede hinsichtlich des Alters, des Geschlechts, der deutschen Staatsangehörigkeit und des Beschäftigungsverhältnisses. Bezüglich des Familienstands und der Bildung unterschieden sich die Gruppen dahin gehend, dass sich die Personen der Traumagruppe seltener in einer festen Beziehung befanden sowie vielfältigere Bildungshintergründe aufwiesen. Die beschriebenen Unterschiede stellen kleine Effekte dar $(\omega<0,3)$ [26]. Eine Übersicht zu den soziodemografischen Merkmalen findet sich in > Tab. 1.

\section{Traumatische Erfahrungen in der Kindheit und Jugend}

Die Probanden der Traumagruppe erreichten im CTQ einen Durchschnittswert von $M=11,79(S D=3,19)$ und wiesen multiple Formen von traumatischen Erfahrungen in der Kindheit und Jugend auf.

- Tab. 1 Charakteristika der Stichprobe.

\begin{tabular}{|c|c|c|c|c|c|}
\hline & $\begin{array}{c}\text { Traumagruppe } \\
\qquad n=61\end{array}$ & $\begin{array}{c}\text { Kontrollgruppe } \\
\quad n=52\end{array}$ & Prüfgröße ${ }^{a}$ & $p$-Wert ${ }^{b}$ & $\begin{array}{l}\text { Effekt- } \\
\text { größe }\end{array}$ \\
\hline \multicolumn{6}{|l|}{ Soziodemografische Merkmale } \\
\hline Alter (M/SD) & $37,8(14,1)$ & $33,3(13,7)$ & $t(111)=1,724$ & 0,088 & - \\
\hline Geschlecht - weiblich (N/\%) & $45(73,3)$ & $34(65,4)$ & $\chi^{2}\left(1, N^{2}=113\right)=0,938$ & 0,333 & - \\
\hline Staatsangehörigkeit - deutsch (N/\%) & $50(82,0)$ & $46(88,5)$ & $\chi^{2}(1, N=113)=0,926$ & 0,336 & - \\
\hline Feste Beziehung (N/\%) & $35(57,4)$ & $40(76,9)$ & $\chi^{2}(1, N=113)=4,352$ & $0,037^{*}$ & $\omega=0,20$ \\
\hline Abitur (N/\%) & $48(78,7)$ & $49(94,2)$ & $\chi^{2}(1, N=113)=5,579$ & $0,018^{*}$ & $\omega=0,22$ \\
\hline Beschäftigungsverhältnis ${ }^{d}$ (N/\%) & $57(93,4)$ & $49(94,2)$ & $\chi^{2}(1, N=113)=0,030$ & 0,862 & - \\
\hline \multicolumn{6}{|l|}{ Psychische Belastung } \\
\hline BDI-II (M/SD) & $5,08(5,52)$ & $1,81(2,83)$ & $\begin{array}{c}t(92)=4,052^{e} \\
z=4,017^{f}\end{array}$ & $0,000^{* * *}$ & $d=0,75$ \\
\hline SCL-27 & $0,35(0,29)$ & $0,14(0,18)$ & $\begin{array}{c}t(102)=4,647^{e} \\
z=4,870^{f}\end{array}$ & $0,000^{* * *}$ & $d=0,84$ \\
\hline ADP-IV & $168,56(44,92)$ & $126,42(24,28)$ & $t(95)=6,323^{e}$ & $0,000^{* * *}$ & $d=1,17$ \\
\hline
\end{tabular}

a $t$-Test, $\chi \chi^{2}$-Test oder U-Test. b Signifikanzniveau $p<0,05 .{ }^{c} d$ oder $\omega$; ${ }^{d}$ Bezieht sich auf derzeitige Ausbildung, Studium oder Beruf. e $t$-Test für heterogene Varianzen aufgrund verletzter Varianzhomogenität. ${ }^{\mathrm{f}}$ Mann-Whitney-U-Test aufgrund verletzter Normalverteilungsannahme. $N=113 .{ }^{*} p<0,05,{ }^{* * *} p<0,001$ 
Fast $70 \%$ der Probanden der Traumagruppe haben mindestens 2 verschiedene Formen von Missbrauchs- oder Vernachlässigungserfahrungen in mäßiger bis extremer Ausprägung in der Kindheit und Jugend erfahren. Insgesamt berichteten knapp $67 \%$ der Traumagruppe sexuelle Missbrauchserfahrungen, $79 \%$ von emotionalem Missbrauch, 51 \% von körperlichem Missbrauch, 82 \% von emotionaler und fast $70 \%$ von körperlicher Vernachlässigung in der Kindheit und Jugend. Es handelte sich bei den Personen der Traumagruppe um eine schwer und multipel traumatisierte Stichprobe. - Abb. 1 zeigt die Verteilung von traumatischen Erfahrungen in der Kindheit und Jugend in der Traumagruppe.

\section{Psychische Belastung}

Die Traumagruppe berichtete eine signifikant höhere Belastung in den psychopathologischen Maßen (BDI-II, SCL-27, ADP-IV) im Vergleich zur Kontrollgruppe. Hierbei fiel der Effekt für den BDI-II moderat $(d=0,75)$ und für die SCL-27 und den ADP-IV groß aus $(d>0,84)$. Trotz der Unterschiede bewegten sich beide Gruppen in einem gesunden Normbereich: Die Mittelwerte des BDI-II sowie der SCL-27 unterschritten die in der Literatur beschriebenen Cutoff-Werte, die klinisch relevante psychopathologische Auffälligkeiten indizieren [23, 24]. Auch für den Gesamtscore des ADP-IV lagen die Mittelwerte in beiden Gruppen im durchschnittlichen Bereich verglichen mit Normwerten aus einer Allgemeinbevölkerungsstichprobe [27]. Nähere Angaben zu den Gruppenunterschieden können $\triangleright$ Tab. 1 entnommen werden.

\section{Der habituelle Einsatz der ER-Strategien}

Bei der Analyse des habituellen Einsatzes der ER-Strategien zwischen den beiden Untersuchungsgruppen zeigten sich signifikante Unterschiede bei den spezifischen Strategien Rumination $(t(111)=2,42, p=0,017, d=0,45)$ und Problemlösen $(t(111)=2,18, p=0,031, d=0,42)$. Die Traumagruppe berichtete häufiger den Einsatz von Rumination und seltener den von Problemlösen als die Kontrollgruppe. Die beobachteten Effekte fielen klein aus $(d<0,50)$. Außerdem unterschieden sich beide Gruppen signifikant dahingehend, dass die Traumagruppe die verhaltensorientierte Strategie Ablenkung typischerweise häufiger verwendet als die Kontrollgruppe $(t(111)=2,50, p=0,014, d=0,48)$. Bezüglich der typischen Nutzung der weiteren ER-Strategien ergaben sich keine signifikanten Unterschiede zwischen den Gruppen. Schließlich erreichten die berichteten Unterschiede nach Anwendung der Bonferroni-Korrektur keine statistische Relevanz. - Abb. 2 zeigt die Verteilung zum habituellen Einsatz der ER-Strategien vor der Bonferroni-Korrektur.

\section{Psychopathologische Belastung als Kovariate}

Um jeweils für den Einfluss des BDI-II, der SCL-27 und des ADP-IV zu kontrollieren, erfolgte die Berechnung von ANCOVAs für Gruppenvergleiche der ER-Strategien Rumination, Problemlösen und Ablenkung ( $\triangleright$ Tab. 2). Unter Kontrolle der SCL-27 waren bei der Strategie Problemlösen signifikante Unterschiede zu beobachten. Diese erreichten jedoch nach Anwendung der Bonferroni-Korrektur keine statistische Signifikanz.

\section{Diskussion}

Die vorliegende Studie untersucht und vergleicht erstmals die habituelle Nutzung spezifischer und verhaltensorientierter ER-Strategien bei einer kaum berücksichtigten Population von psychisch gesunden erwachsenen Personen mit und ohne traumatische Erfahrungen in der Kindheit und Jugend. Ziel war zu überprüfen, ob eine funktionale ER für einen resilienten Entwicklungsverlauf und damit für die Aufrechterhaltung der psychischen Gesundheit nach frühen traumatischen Erfahrungen bedeutsam ist. Aufgrund der inkonsistenten Studienlage wurde eine der folgenden Varianten erwartet: Die Traumagruppe weist verglichen mit der Kontrollgruppe eine funktionalere, eine ähnlich ausgeprägte oder eine dysfunktionalere habituelle Nutzung der ER-Strategien auf.

Psychisch gesunde Personen mit traumatischen Erfahrungen in der Kindheit und Jugend können als Hochrisikostichprobe für Psychopathologie angesehen werden [3], was durch die Ergebnisse der vorliegenden Arbeit gestützt wird. Nahezu $70 \%$ der Probanden

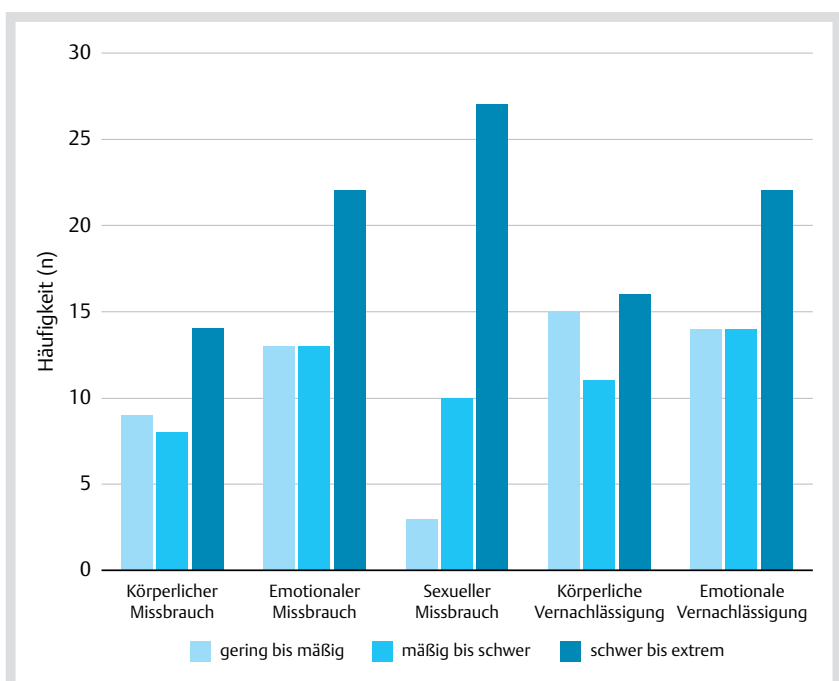

- Abb. 1 Formen und Schweregrad der traumatischen Erfahrungen in der Kindheit und Jugend (Traumagruppe). $N=61$. Kategorienbildung nach Cut-Off-Werten des CTQ (Bernstein \& Fink, 1998).

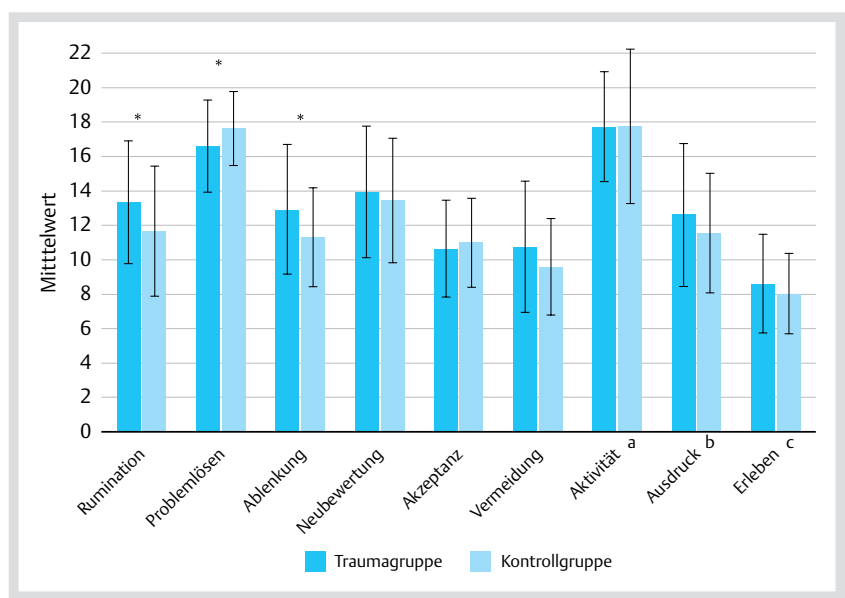

Abb. 2 Habitueller Einsatz der ER-Strategien. $N=113$. Erhoben mit H-FERST. Die Fehlerbalken bilden Standardabweichungen. a Aktivität

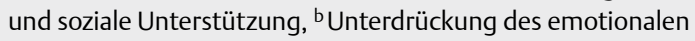
Ausdrucks, ' Unterdrückung des emotionalen Erlebens, ${ }^{*} p<0,05$. 
- Tab. 2 Kovarianzanalysen (ANCOVAs) von Gruppenunterschieden in den ER-Strategien Rumination, Problemlösen und Ablenkung unter Kontrolle der psychopathologischen Belastung (BDI-II, SCL-27 und ADP-IV).

\begin{tabular}{|c|c|c|c|c|c|}
\hline & \multirow{2}{*}{$\begin{array}{l}\text { Traumagruppe } \\
\begin{array}{c}n=61 \\
M(S D)\end{array}\end{array}$} & \multirow{2}{*}{$\begin{array}{l}\text { Kontrollgruppe } \\
\qquad \begin{array}{c}n=52 \\
M(S D)\end{array}\end{array}$} & \multicolumn{3}{|c|}{ ANCOVA } \\
\hline & & & Haupteffekt der Gruppe & $p$-Wert a & $\eta^{2}$ \\
\hline \multicolumn{6}{|c|}{ Rumination } \\
\hline BDI-II & $13,33(3,57)$ & $11,65(3,78)$ & $F(110)=2,981$ & 0,087 & - \\
\hline $\mathrm{SCL}-27^{\mathrm{b}}$ & $13,33(3,57)$ & $11,73(3,79)$ & $F(109)=0,473$ & 0,493 & - \\
\hline ADP-IV & $13,33(3,57)$ & $11,65(3,78)$ & $F(110)=0,026$ & 0,872 & - \\
\hline \multicolumn{6}{|c|}{ Problemlösen } \\
\hline BDI-II & $16,61(2,68)$ & $17,62(2,14)$ & $F(110)=3,290$ & 0,072 & - \\
\hline $\mathrm{SCL}-27^{\mathrm{b}}$ & $16,61(2,68)$ & $17,59(2,16)$ & $F(109)=4,038$ & $0,047^{*}$ & - \\
\hline ADP-IV & $16,61(2,68)$ & $17,62(2,14)$ & $F(110)=1,592$ & 0,210 & - \\
\hline \multicolumn{6}{|c|}{ Ablenkung } \\
\hline BDI-II & $12,92(3,78)$ & $11,31(2,89)$ & $F(110)=2,668$ & 0,105 & - \\
\hline $\mathrm{SCL}-27^{\mathrm{b}}$ & $12,92(3,78)$ & $11,33(2,91)$ & $F(109)=2,429$ & 0,122 & - \\
\hline ADP-IV & $12,92(3,78)$ & $11,31(2,89)$ & $F(110)=0,003$ & 0,954 & - \\
\hline
\end{tabular}

der Traumagruppe erlebten in ihrer Kindheit oder Jugend multiple traumatische Erfahrungen moderaten bis schweren Ausmaßes. Zudem wies die Traumagruppe in den Selbstratings (BDI-II, SCL-27, ADP-IV) eine höhere psychische Belastung auf als die Kontrollgruppe, war aber dennoch psychopathologisch als unauffällig einzustufen. Ähnliche Befunde wurden bereits berichtet [23, 27, 28]. Durch eine ausführliche psychopathologische Untersuchung (SKID-I) konnte gesichert werden, dass die Probanden der Traumagruppe die Kriterien psychischer Gesundheit (im Sinne einer Abwesenheit manifester psychischer Störungen sowohl aktuell als auch über die bisherige Lebensspanne hinweg) erfüllten und insofern als resilient bezeichnet werden können. Bemerkenswert ist, dass die Traumagruppe dennoch eine höhere subjektive psychische Belastung im Vergleich zu nicht traumatisierten psychisch gesunden Personen aufwies. Dieser Befund kann dahingehend interpretiert werden, dass die traumatischen Erfahrungen in der Kindheit und Jugend nicht „spurlos“ an den Probanden der Traumagruppe vorbeigegangen zu sein scheinen, sondern sie durchaus höher belastet sind.

Die beiden Gruppen unterschieden sich im habituellen Einsatz der ER-Strategien Rumination, Problemlösen und Ablenkung signifikant voneinander. Allerdings ist durch die erhöhte subjektive psychische Belastung der Traumagruppe eine Verzerrung der Ergebnisse nicht auszuschließen. Diese Überlegung wurde durch das kleine Ausmaß der Effekte $(d<0,50)$ gestützt. Unter Kontrolle dieses möglichen konfundierenden Faktors verschwanden die beobachteten Effekte des Gruppenunterschieds bei allen 3 ER-Strategien $\left(\eta^{2}=0,00\right)$. Zusammenfassend unterschieden sich die beiden Gruppen hinsichtlich der habituellen Nutzung von adaptiven und maladaptiven ER-Strategien nicht bedeutsam voneinander. Die Personen der Traumagruppe zeigten demnach trotz erlebter trauma- tischer Erfahrungen in der Kindheit und Jugend sowie höherer subjektiver psychischer Belastung ein vergleichbares ER-Profil wie die Kontrollgruppe. Dies bestätigt teilweise die Befunde von Lang, Kotchoubey, Frick, et al. [29], die ebenso keine Unterschiede im selbstberichteten Einsatz von Neubewertung zwischen traumatisierten und nicht traumatisierten gesunden Personen beobachteten.

Diese Befunde lassen sich durch den Zusammenhang der untersuchten ER-Strategien und der An- bzw. Abwesenheit von Psychopathologie erklären, wonach der Einsatz adaptiver ER-Strategien mit weniger psychopathologischen Symptomen assoziiert ist (vgl. [14]). Aber sowohl durch das vorliegende querschnittliche Studiendesign als auch durch die bisher begrenzten längsschnittlichen Untersuchungen bleibt die kausale Beziehung zwischen ER und Psychopathologie ungeklärt (vgl. [14, 30]). So wäre es zum einen denkbar, dass das mit einer psychisch gesunden Kontrollgruppe ohne Traumatisierung vergleichbare ER-Profil der Traumagruppe durch deren ohnehin gegebene psychische Gesundheit erklärt werden kann. Demzufolge hätte das Erleben traumatischer Erfahrungen keinen Einfluss auf die ER bei Personen der Traumagruppe, da sich aufgrund ihrer psychischen Gesundheit ein funktionaler Einsatz von adaptiven und maladaptiven ER-Strategien findet. Zum anderen könnte angesichts des gegebenen psychopathologischen Risikos vor dem Hintergrund traumatischer Erfahrungen in der Kindheit und Jugend und einer subjektiv höheren psychischen Belastung auch davon ausgegangen werden, dass die Traumagruppe aufgrund ihrer Fähigkeit zum funktionalen Einsatz von adaptiven und maladaptiven ER-Strategien psychisch gesund geblieben ist. Damit könnte einer funktionalen und effektiven ER eine bedeutsame Rolle für die Aufrechterhaltung der psychischen Gesundheit nach dem Erleben früher traumatischer Erfahrungen zukommen [31]. Allerdings ist bei der Interpretation der Befunde die Komplexität des Zusam- 
menspiels verschiedener Resilienzfaktoren zu beachten, wie einer Vielzahl psychologischer, biologischer, interpersoneller und soziokultureller Faktoren [6]. Unter Berücksichtigung dieser Interaktionen, könnte eine funktionale Nutzung von adaptiven und maladaptiven ER-Strategien bedeutsam sein für die resiliente Entwicklung von erwachsenen Individuen nach dem Erleben traumatischer Erfahrungen in der Kindheit und Jugend. Dieser Erklärung folgend könnte es sein, dass die Probanden der Traumagruppe einen viel höheren Aufwand betreiben, so z. B. negative Emotionen noch effektiver regulieren, um angesichts der höheren subjektiven psychischen Belastung durch die traumatischen Erfahrungen in der Kindheit und Jugend die psychische Gesundheit zu erhalten.

Einschränkend ist anzumerken, dass in der vorliegenden Studie keine traumatisierte Gruppe mit psychischen Erkrankungen untersucht wurde, sodass die obigen Überlegungen entsprechend der Relevanz für eine resiliente Entwicklung, nicht aber für eine psychopathologische Entwicklung zu interpretieren sind. Weiterhin scheint die vorliegende Stichprobe einer Selektivität hinsichtlich des Bildungsniveaus zu unterliegen (über $80 \%$ der Probanden verfügen über eine Hochschulreife), sodass eine Verzerrung der Ergebnisse dahingehend nicht auszuschließen ist. Gleichzeitig gilt ein hohes akademisches Bildungsniveau als Schutzfaktor nach frühen traumatischen Erfahrungen [32], sodass Wechselwirkungen zwischen diesem sozioökonomischen Merkmal und ER-Prozessen denkbar sein könnten. Weitere Einschränkungen der Untersuchung sind der Einsatz von subjektiven Selbstbeschreibungsfragebögen, da erinnerungsbedingte und stimmungsinduzierte Verzerrungseffekte nicht ausgeschlossen werden können. So können beispielsweise durch die retrospektive Erhebung der traumatischen Erfahrungen in der Kindheit und Jugend mittels CTQ Fehleinschätzungen durch Erinnerungsverzerrungen, insbesondere bei lang zurückliegenden Traumata im Kindes- und Jugendalter, vorliegen [33]. Um diesen Verzerrungseffekten entgegen zu wirken, wurden die Angaben des CTQ in der vorliegenden Untersuchung im Screening um eine zusätzliche Exploration ergänzt. Weiterhin ist die Erhebung der habituellen Nutzung von ER-Strategien durch den Fragebogen H-FERST in ihrer Validität und Reliabilität insofern limitiert, dass wichtige Kontextfaktoren sowie unbewusste Anteile der ER vernachlässigt werden. Insbesondere bei adaptiven Strategien scheinen der Kontext und die Flexibilität ihres Einsatzes von großer Bedeutung für deren Funktionalität bezüglich Psychopathologie zu sein [34]. Darüber hinaus ist die Generalisierbarkeit der Befunde auf die ER nach Trauma-Typ-I Erfahrungen eingeschränkt.

Unter Berücksichtigung der genannten Limitationen wären in zukünftigen Studien längsschnittliche und prospektive Untersuchungen in Kombination mit einer klinischen Stichprobe wünschenswert, um fundierte Aussagen über die kausale Beziehung zwischen traumatischen Erfahrungen im Kindes- und Jugendalter, ER und der psychischen Gesundheit im Erwachsenenalter zu treffen [35].

Abschließend beinhalten die Ergebnisse unter der Berücksichtigung der aufgeführten Limitationen Implikation für Präventionsmaßnahmen zur Aufrechterhaltung der psychischen Gesundheit nach dem Erleben traumatischer Erfahrungen in der Kindheit und Jugend: Vor dem Hintergrund traumatischer Erfahrungen in der Kindheit und Jugend könnte eine funktionale Nutzung von adaptiven und maladaptiven ER-Strategien zur Aufrechterhaltung der psy- chischen Gesundheit beitragen. Gleichzeitig wird so der Entwicklung von ER-Defiziten vorgebeugt, die sich wiederum begünstigend auf eine psychopathologische Entwicklung auswirken können. Demnach könnte nach dem Erleben traumatischer Erfahrungen in der Kindheit und Jugend eine gezielte Nachsorge in Form eines ERTrainings zum Erlernen adaptiver ER-Strategien hilfreich sein, wie z. B. im Rahmen des gruppentherapeutischen Programms Gefühle im Griff [36]. Dadurch können Betroffenen für die Relevanz emotionaler Prozesse sensibilisiert werden, was sich auf die Entwicklung einer funktionalen und damit effektiven ER auswirken könnte.

\section{FAZIT FÜR DIE PRAXIS}

Das Erleben traumatischer Erfahrungen in der Kindheit und Jugend gilt als Risikofaktor für spätere psychopathologische Symptome. Den Ergebnissen der vorliegenden Studie zufolge könnte eine funktionale Emotionsregulation (ER) einen resilienten Entwicklungsverlauf fördern. Demnach erscheint nach dem Erleben früher traumatischer Erfahrungen der präventive Einsatz eines ER-Trainings zum Erlernen adaptiver ER-Strategien sinnvoll. So werden die Betroffenen für die Relevanz emotionaler Prozesse sensibilisiert und in ihrer Fähigkeit gestärkt mit einer funktionalen ER der Entwicklung psychopathologischer Symptome entgegenzuwirken.

Interessenkonflikt

Die Autoren geben an, dass kein Interessenkonflikt besteht.

Literatur

[1] Iffland B, Brahler E, Neuner $F$ et al. Frequency of child maltreatment in a representative sample of the German population. BMC Public Health 2013; 13: 980

[2] Terr LC. Childhood traumas: an outline and overview. Am J Psychiatry 1991; 148: 10-20

[3] Kessler RC, McLaughlin KA, Green JG et al. Childhood adversities and adult psychopathology in the WHO World Mental Health Surveys. $\mathrm{Br}$ J Psychiatry 2010; 197: 378-385

[4] McGloin JM, Widom CS. Resilience among abused and neglected children grown up. Dev Psychopathol 2001; 13: 1021-1038

[5] Ungar M. Practitioner Review: Diagnosing childhood resilience - a systemic approach to the diagnosis of adaptation in adverse social and physical ecologies. J Child Psychol Psychiatry 2015; 56: 4-17

[6] Cicchetti D. Annual Research Review: Resilient functioning in maltreated children - past, present, and future perspectives. J Child Psychol Psychiatry 2013; 54: 402-422

[7] Kim J, Cicchetti D. Longitudinal pathways linking child maltreatment, emotion regulation, peer relations, and psychopathology. J Child Psychol Psychiatry 2010; 51: 706-716

[8] Cheetham A, Allen NB, Yucel M et al. The role of affective dysregulation in drug addiction. Clin Psychol Rev 2010; 30: 621-634

[9] Kashdan TB, Rottenberg J. Psychological flexibility as a fundamental aspect of health. Clin Psychol Rev 2010; 30: 865-878

[10] Runtz MG, Schallow JR. Social support and coping strategies as mediators of adult adjustment following childhood maltreatment. Child Abuse Negl 1997; 21: 211-226 
[11] Gross J]. The emerging field of emotion regulation: An integrative review. Rev Gen Psychol 1998; 2: 271-299

[12] Barnow S, Löw CA, Dodek A et al. Managing emotions - emotions under control. Psychother Psych Med 2014; 64: 284-289

[13] Campbell-Sills L, Barlow DH. Incorporating emotion regulation into conceptualizations and treatments of anxiety and mood disorders. In: Gross J], (Hrsg.). Handbook of emotion regulation New York. NY, US: Guilford Press; 2007: 542-559

[14] Aldao A, Nolen-Hoeksema S, Schweizer S. Emotion-regulation strategies across psychopathology: A meta-analytic review. Clin Psychol Rev 2010; 30: 217-237

[15] Holl J, Wolff S, Schumacher $M$ et al. Substance use to regulate intense posttraumatic shame in individuals with childhood abuse and neglect. Dev Psychopathol 2016; 1-13

[16] Cloitre M, Miranda R, Stovall-McClough KC et al. Beyond PTSD: emotion regulation and interpersonal problems as predictors of functional impairment in survivors of childhood abuse. Behav Ther 2005; 36:119-124

[17] Simon VA, Smith E, Fava N et al. Positive and negative posttraumatic change following childhood sexual abuse are associated with youths' adjustment. Child Maltreat 2015; 20: 278-290

[18] Klanecky AK, McChargue DE. Emotional reactivity across individuals with varying trauma and substance dependence histories. Ment Health Subst Use 2009; 2: 191-202

[19] Bernstein DP, Fink L. Childhood trauma questionnaire: A retrospective self-report manual. San Antonio, TX: The Psychological Corporation; 1998

[20] Wingenfeld K, Spitzer C, Mensebach C et al. The German version of the Childhood Trauma Questionnaire (CTQ): preliminary psychometric properties. Psychother Psychosom Med Psychol 2010; 60: 442-450

[21] Wittchen H-U, Zaudig M, Fydrich T., (Hrsg.). Strukturiertes klinisches Interview für DSM-IV: SKID; eine deutschsprachige, erweiterte Bearbeitung der amerikanischen Originalversion des SCID Aufl. Göttingen [u. a.]: Hogrefe; 1997

[22] Hautzinger M, Keller F, Kühner C. Beck Depressions-Inventar : BDI II. Revision. Revision. Aufl. Frankfurt am Main: Harcourt Test Services; 2006

[23] Kühner C, Bürger C, Keller F et al. Reliability and validity of the Revised Beck Depression Inventory (BDI-II). Results from German samples. Nervenarzt 2007; 78: 651-656
[24] Hardt J, Egle UT, Kappis B et al. Die Symptom-Checkliste SCL-27 Ergebnisse einer deutschen Reprasentativbefragung. Psychother Psych Med 2004; 54: 214-223

[25] Doering S, Renn D, Hofer $S$ et al. Validierung der deutschen Version des Fragebogens zur Erfassung von DSM-IV Personlichkeitsstorungen (ADP-IV). Zeitschrift für Psychosomatische Medizin und Psychotherapie 2007; 53: 111-128

[26] Cohen J. Statistical power analysis for the behavioral sciences. 2 ed. Hillsdale, NJ [u. a.]: Erlbaum; 1988

[27] Renn D. Fragebogen zur Erfassung von Persönlichkeitsstörungen: ADP-IV: Universität Innsbruck 2006

[28] Hardt J, Egle UT, Brähler E. Die Symptom-Checkliste-27 in Deutschland. Psychother Psych Med 2006; 56: 276-284

[29] Lang S, Kotchoubey B, Frick C et al. Cognitive reappraisal in traumaexposed women with borderline personality disorder. Neurolmage 2012; 59: 1727-1734

[30] Barnow S. Emotionsregulation und Psychopathologie. Psychologische Rundschau 2012; 63: 111-124

[31] Choi JY, Choi YM, Gim MS et al. The effects of childhood abuse on symptom complexity in a clinical sample: Mediating effects of emotion regulation difficulties. Child Abuse Negl 2014, doi:10.1016/j. chiabu.2014.04.016

[32] Edmond T, Auslander W, Elze D et al. Signs of resilience in sexually abused adolescent girls in the foster care system. J Child Sex Abus 2006; 15: 1-28

[33] Hardt J. Retrospektive Erfassung von Kindheitsbelastungen bei Erwachsenen. In: Egle UT, Hoffmann SO, Joraschky P, (Hrsg.). Sexueller Missbrauch, Misshandlung, Vernachlässigung: Erkennung, Therapie und Prävention der Folgen früher Stresserfahrungen. Stuttgart: Schattauer; 2005: 222-246

[34] Aldao A. The future of emotion regulation research: capturing context. Perspect Psychol Sci 2013; 8: 155-172

[35] Bardeen JR, Kumpula M], Orcutt HK. Emotion regulation difficulties as a prospective predictor of posttraumatic stress symptoms following a mass shooting. J Anxiety Disord 2013; 27: 188-196

[36] Barnow S. Gefühle im Griff!: Wozu man Emotionen braucht und wie man sie reguliert. Berlin, Heidelberg: Springer; 2014 